\title{
A method for the quantitation of small intestinal biopsy specimens
}

\author{
M. S. DUNNILL AND R. WHITEHEAD \\ From the Gibson Laboratories, Radcliffe Infirmary, Oxford
}

SYNOPSIS A simple method is described for the quantitative examination of small intestinal mucosal biopsies involving the determination of an index related to the surface-to-volume ratio of the mucosa. The method is of value in distinguishing minor degrees of villous abnormality and will prove useful in comparing serial changes in a given patient. It will also allow comparisons to be made between biopsies taken in different medical centres.

Small intestinal biopsy is an essential procedure in the investigation of all forms of malabsorption. On ordinary light microscopy severe or specific abnormalities of the mucosa present little difficulty in interpretation, but non-specific minor degrees of atrophy are notoriously difficult to assess even for the experienced. There is a particular need for a quantitative method of examination so that these minor changes can be accurately recorded and this is especially true when serial biopsies from the same patient are being studied. Such a method if easily standardized would also allow comparison between material obtained at different centres. Earlier attempts at quantitation (Rubin, Brandborg, Phelps, Taylor, Murray, Stemler, Howry, and Volwiler, 1960; Thurlbeck, Benson, and Dudley, 1960; Madanagopalan, Shiner, and Rose, 1965; Stewart, Pollock, Hoffbrand, Mollin, and Booth, 1967) were based on linear micrometer measurement of mucosal thickness, crypt length, and villous height or the use of intersecting lines to assess villous surface area. They are to be criticized because accurate measurement of the length of villi which are atrophic, distorted, bifurcate, or not in an upright position is difficult, and the decision as to where a villus ends and a crypt begins is arbitrary and introduces an individual subjective element. Mucosal biopsies which do not include the muscularis mucosae and, especially those which have been stretched longitudinally, will show separation or spreading of villi accompanied by a decrease in height; simple linear measurements of villous height and crypt length and the use of intersecting lines to assess villous surface area without relating this to volume will give an erroneous assessment of the mucosa. It is doubtful if these Received for publication 14 September 1971. inherently inaccurate methods give any better idea of minor villous abnormalities than a simple visual assessment by an experienced observer, and some workers (Rubin and Dobbins, 1965) have resorted to 'the method of blind review'.

Several morphometric methods other than direct linear measurement are, however, available for the measurement of tissue components in histological sections (Dunnill, 1968). All these methods depend on (1) adequate sampling and (2) the structures that are measured being randomly distributed. Neither of these conditions apply in the intestinal biopsy where great care is taken to ensure that the biopsy is embedded in such a position that histological sections can be cut perpendicular to the luminal surface.

Chalkley, Cornfield, and Park (1949) developed a method for the determination of surface volume ratios of randomly distributed three-dimensional bodies within a structure from examination of thin, ie, two-dimensional, sections of the structure. The method combined the point counting principle (Chalkley, 1943) for volume determination with the linear intercept principle (Short, 1950) for surface area measurement. They used a system consisting of a series of short lines of equal length, 1 , which were placed at random on sections of the material containing the bodies under examination. The number of times, $h$, the end points of these lines fell within, or 'hit' the structures was counted. This is proportional to the volume of the structures. The number of intersections, or 'cuts', c, made by the lines with the surface of the bodies, ie, the perimeter of the bodies as seen in thin histological sections, was recorded. This is proportional to the surface area of the bodies. It can be shown that providing a large 
number of lines is used then the surface to volume ratio is given by:

$$
\frac{S}{V}=\frac{4 c}{1 h}
$$

An adaptation of this method, while not giving absolute value for the surface volume ratio, due to the fact that the intestinal mucosal biopsy is not randomly orientated and sectioned, will at least allow comparisons to be made between biopsies and will give an accurate and reproducible indication of the degree of villous atrophy. In their original paper Chalkley et al (1949) used the reciprocal of this expression and determined the volume-to-surface ratio. In small intestinal studies where the main concern is with the villous surface an index of the surface-to-volume ratio is likely to prove of more value.

\section{Materials and Methods}

Peroral small intestine biopsies were obtained using a Crosby capsule. They were fixed in $10 \%$ formal saline, dehydrated, cleared, and embedded in paraffin wax.
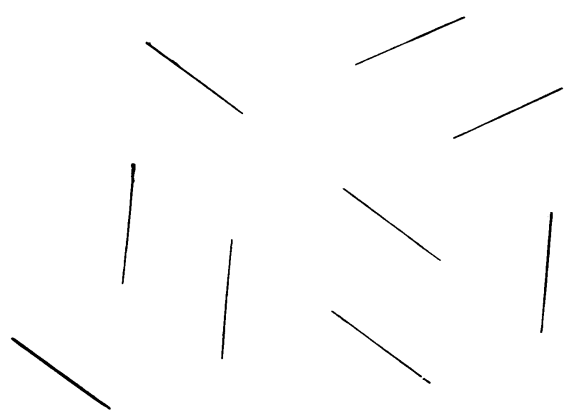

Fig. 1a.
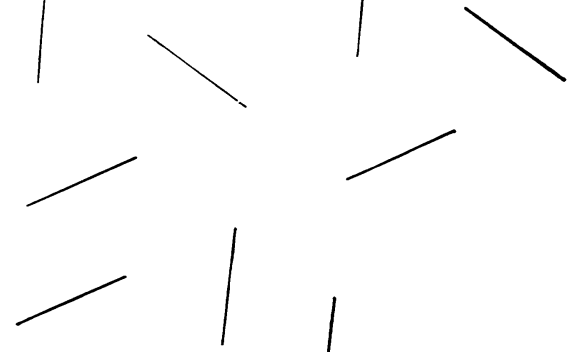

Fig. 1 a The template of 15 lines of equal length drawn according to specifications of Weibel (1963).

Fig. 1 b The template superimposed on a normal intestinal biopsy. The end points of the lines lying on the mucosa are counted as hits. The places where the lines cut the mucosal surface are counted as cuts.
Embedding was carried out with the aid of a dissecting microscope to ensure that the histological sections were cut perpendicular to the mucosa. Sections were cut at $4 \mu$ and stained by haematoxylin and eosin or by the periodic-acid Schiff method. A Leitz projector fitted with a Xenon lamp was used to project the sections onto a piece of Bristol board on which was drawn a series of lines of equal length conforming to the pattern shown in Figure 1. This is the template devised by Weibel (1963) and is composed of 15 lines of equal length connecting the vertices of a regular hexagonal point network. Weibel states that this ensures that the end points are evenly distributed and avoids any bias.

The magnification was kept constant throughout this investigation and was such that the length of each line cast on the section was $1.4 \times 10^{-2} \mathrm{~cm}$. The section was cast at random on the template and the number of times, $c$, the lines cut the mucosal surface counted and recorded. The number of end points of the lines, $\mathrm{h}$, falling on the tissue between the mucosal cells and the muscularis mucosae was also recorded. The greater the number of fields cast on the template the more accurate will be the final result but in practice it was found to be sufficient if the

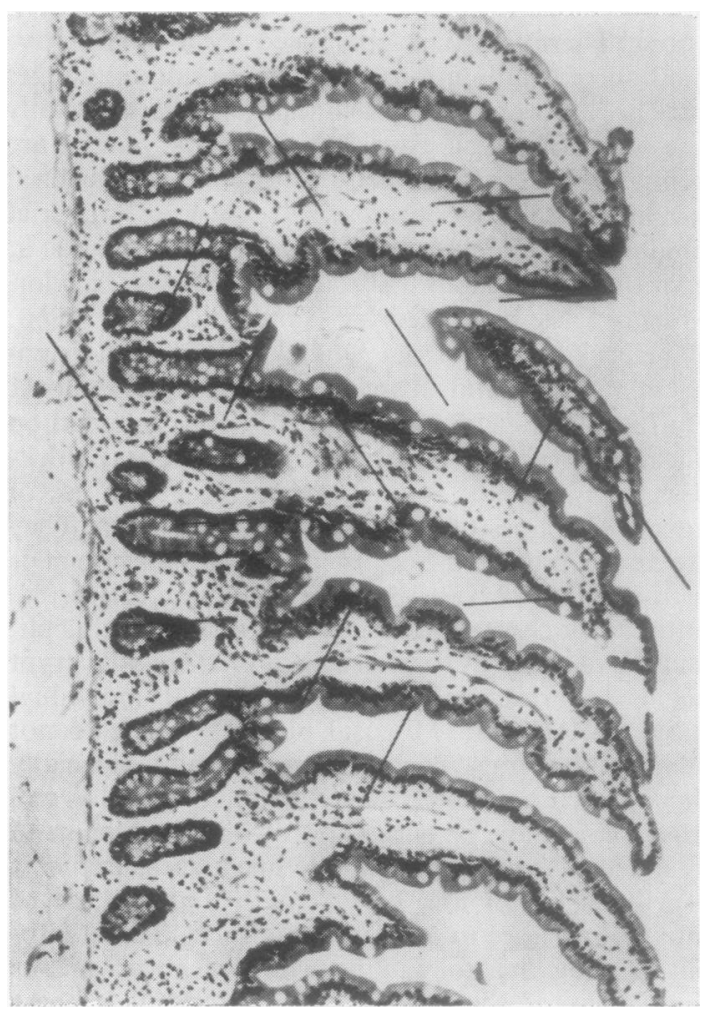

Fig. 1b. 
value for $h$ was approximately 200 . If the biopsy specimen is a good-sized one, it is possible to superimpose the grid on more than one field in a single section; if this is so, it is usually sufficient to make counts on two or three sections. If the biopsy specimen is small, a larger number of serial sections must be examined, perhaps up to six or eight.

The ratio $c$ : lh will then give an index of the complexity of the villous pattern and of the surfaceto-volume ratio though, due to the standard method of orientating the specimens, it is not possible to calculate the absolute ratio by the method of Chalkley et al (1949). It is important to include the length of the line, 1 , in order that values for the ratio $\mathrm{c}: \mathrm{lh}$ can be compared between different centres. The simple ratio of 'hits' to 'cuts' will, of course, alter with the length of line used.

A further important piece of information can be obtained from these data. Providing the magnification is kept constant the mean number of hits per field on the mucosa, $\bar{h}$, for each specimen will be proportional to the mucosal volume. It is thus possible to compare not only the state of the villi but also the mucosal volumes between specimens.

In order to test this method of assessing intestinal mucosal biopsies the following material was chosen. (1) Eleven biopsies from normal patients. (2) Six biopsies from patients with the malabsorption syndrome in which the appearances when assessed subjectively varied between one bordering on the normal to one showing mild villous atrophy. The biopsies were from two patients with treated gluten-sensitive enteropathy, one patient with giardiasis, and three with evidence of the malabsorption syndrome in whom a definite diagnosis was not made. (3) Twelve biopsies from cases of untreated gluten-sensitive enteropathy were assessed histologically as showing hyperplasia of the crypts, severe partial villous atrophy, or total absence of villi.
Results

The results for each set of cases are shown in Table I. In the normal subjects the mean value of the ratio c : lh was 46.0 (sd 12.0) whereas in the biopsies labelled as atrophic the mean value was $8 \cdot 4$ (sd 3.5). This is a highly significant difference $(t=10.4$; $P<0.001$ ). More interesting, however, are the six cases in which some difficulty was experienced in arriving at a diagnosis by conventional subjective histological examination of the biopsy. In this group the mean value for the ratio $c$ : : lh was 23.7 (sd 2.9) and there was no overlap with the normal mucosa; indeed the difference between the two groups was statistically highly significant $(t=4.41 ; P<0.001)$. It would seem, therefore, that the method is a valuable one for detecting minor degrees of villous abnormality.

Although the biopsies of groups 2 and 3 show atrophy of villi, the actual volume of the mucosa is not diminished and may even be increased. This is shown by the fact that there is no significant difference $(t=2.07 ; 0.1>P>0.05)$ between the mean number of hits per field, $\bar{h}$, in the normal mucosa, 16.3 (sd 4.1) and the cases with villous atrophy, $19 \cdot 2$ (sd 2.6). This is due to the fact that notwithstanding the atrophy of villi the crypts elongate and the lamina propria probably also increases in volume, a point which could be resolved by a further quantitative study.

\section{Discussion}

The introduction of terms to denote histological appearances when these are based on rather vague and loosely defined subjective impressions is of little value. This is particularly true for lesions which are of a progressive nature and show a range varying from normal to one which is grossly abnormal. Such

\begin{tabular}{|c|c|c|c|c|c|c|c|c|}
\hline \multicolumn{3}{|c|}{ Normal Subjects } & \multicolumn{3}{|c|}{ Intermediate Cases } & \multicolumn{3}{|c|}{ Atrophic Cases } \\
\hline $\begin{array}{l}\text { Case } \\
\text { No. }\end{array}$ & $\begin{array}{l}c: l h \\
(\mathrm{~cm})\end{array}$ & $\begin{array}{l}\text { Mean Number of Hits } \\
\text { per Field } h\end{array}$ & $\begin{array}{l}\text { Case } \\
\text { No. }\end{array}$ & $\begin{array}{l}c: l h \\
(\mathrm{~cm})\end{array}$ & $\begin{array}{l}\text { Mean Number of Hits } \\
\text { per Field } \bar{h}\end{array}$ & $\begin{array}{l}\text { Case } \\
\text { No. }\end{array}$ & $\begin{array}{l}c: l h \\
(\mathrm{~cm})\end{array}$ & $\begin{array}{l}\text { Mean Number of Hits } \\
\text { per Field } \boldsymbol{h}\end{array}$ \\
\hline $\begin{array}{r}1 \\
2 \\
3 \\
4 \\
5 \\
6 \\
7 \\
8 \\
9 \\
10 \\
11\end{array}$ & $\begin{array}{l}41.5 \\
52.9 \\
46.7 \\
29.8 \\
74.6 \\
34.4 \\
39.8 \\
37.5 \\
46.9 \\
52.6 \\
49.0\end{array}$ & $\begin{array}{r}8.3 \\
13.4 \\
14.0 \\
19 \cdot 6 \\
16.3 \\
13.7 \\
21 \cdot 3 \\
17 \cdot 7 \\
22.9 \\
16.4 \\
15.8\end{array}$ & $\begin{array}{l}12 \\
13 \\
14 \\
15 \\
16 \\
17\end{array}$ & $\begin{array}{l}23 \cdot 4 \\
22 \cdot 0 \\
26 \cdot 4 \\
24 \cdot 9 \\
18 \cdot 7 \\
26 \cdot 7\end{array}$ & $\begin{array}{l}15.5 \\
13.7 \\
16.1 \\
12.6 \\
19.7 \\
15.8\end{array}$ & $\begin{array}{l}18 \\
19 \\
20 \\
21 \\
22 \\
23 \\
24 \\
25 \\
26 \\
27 \\
28 \\
29\end{array}$ & $\begin{array}{r}5.6 \\
6.6 \\
7.7 \\
8.1 \\
6.7 \\
4.3 \\
10.5 \\
4.9 \\
9.6 \\
10.2 \\
8.4 \\
17.8\end{array}$ & $\begin{array}{l}17.9 \\
22.7 \\
21.6 \\
19.0 \\
22.2 \\
20.0 \\
15.8 \\
22.4 \\
18.4 \\
16.0 \\
17.8 \\
16.8\end{array}$ \\
\hline
\end{tabular}


is the lesion of villous atrophy of the small intestine. The terms 'partial villous atrophy' and 'subtotal villous atrophy' (Shiner and Doniach, 1960), whilst having served a useful purpose in the early days of studies in jejunal morphology, do not adequately allow for an accurate description of all the possible appearances in jejunal biopsies showing atrophic changes (Whitehead, 1971), and whilst the more elaborate subdivisions into groups (Watson, Paton, and Murray, 1965) partially overcomes this defect, they depend too heavily upon imprecise subjective impressions.

No matter how carefully carried out, these methods do not provide reliable means for comparing data obtained in different centres. The present method of examining mucosal biopsies overcomes these difficulties to a large extent, because the $c: l h$ ratio provides an accurate assessment of the villous surface area in relation to mucosal volume although not in absolute terms.

A further interesting point is brought to light when conditions are studied where the volume of the mucosa decreases as well as the surface area. Then the ratio $c:$ lh may not alter significantly from the normal value. This is illustrated by a small series of intestinal biopsies taken from treated and untreated patients with pernicious anaemia (a study reported in full by Peña, Callender, Truelove, and Whitehead, 1971). These biopsies were all examined under the same magnification. The mean value for the ratio $c: l h$ in the untreated cases, $34 \cdot 3$ (sd 5.3), did not differ significantly from the value found in the treated cases, 40.2 (sd 14.9). However, the mean value for the number of hits on the mucosa in the untreated cases, 13.3 (sd 2.0), was significantly less than in the treated cases, $17.6(\mathrm{sd} \mathrm{2.8})(\mathrm{t}=2.87$; $P<0.005$ ), indicating that the mucosal volume was reduced in the former group, but increased after treatment.
In reporting small intestinal biopsy specimens, it would be valuable, and indeed enable results obtained in different centres to be compared, to give the value for $c: l h$ and $\bar{h}$ in the same manner as the clinical biochemist gives a numerical value for the blood sugar or blood urea. It might be argued that the procedure for making these histological measurements is too laborious and time consuming. This is not so. In fact, an individual case rarely takes more than five to 10 minutes.

\section{References}

Chalkley, H. W. (1943). Method for the quantitative morphological analysis of tissues. $J$. nat. Cancer Inst., 4, 47-53.

Chalkley, H. W., Cornfield, J., and Park, H. (1949). A method for estimating volume-surface ratios. Science, 110, 295-297.

Dunnill, M. S. (1968). In Recent Advances in Clinical Pathology, Series V, edited by S. C. Dyke, pp. 401-416. Churchill, London.

Madanagopalan, N., Shiner, M., and Rose, B. (1965). Measurements of small intestinal mucosa obtained by peroral biopsy. Amer. J. Med., 38, 42-53.

Peña, A. S., Callender, S. T., Truelove, S. C., and Whitehead, R. (1971). Small intestinal mucosal abnormalities and disaccharidase activity in pernicious anaemia. (In preparation.)

Rubin, C. E., Brandborg, L. L., Phelps, P. O., Taylor, H. C., Murray, C. V., Stemler, R., Howry, C., and Volwiler, W. (1960). Studies in celiac disease. Gastroenterology, 38, 517-535.

Rubin, C. E., and Dobbins, W. O., III (1965). Peroral biopsy of the small intestine. Gastroenterology, 49, 676-697.

Shiner, M., and Doniach, I. (1960). Histopathologic studies in steatorrhoea. Gastroenterology, 38, 419-440.

Short, R. H. D. (1950). Alveolar epithelium in relation to growth of the lung. Phil. Trans. B., 235, 35-86.

Stewart, J. S., Pollock, D. J., Hoffbrand, A. V., Mollin, D. L., and Booth, C. C. (1967). A study of proximal and distal intestinal structure and absorptive function in idiopathic steatorrhoea. Quart. J. Med., 36, 425-444.

Thurlbeck, W. M., Benson, J. A., Jr, and Dudley, H. R., Jr. (1960). The histopathologic changes of sprue and their significance. Amer. J. clin. Path., 34, 108-117.

Watson, W. C., Paton, E., and Murray D. (1965). Small-bowel disease in rosacea. Lancet, 2, 47-50.

Weibel, E. R. (1963). Principles and methods for the morphometric study of the lung and other organs. Lab. Invest., 12, 131-155.

Whitehead, R. (1971). The interpretation and significance of morphological abnormalities in jejunal biopsies. J. clin. Path., 24, Suppl. (Roy. Coll. Path.), 5, 108-124. 